\title{
Divergent Auditory Nerve Encoding Deficits Between Two Common Etiologies of Sensorineural Hearing Loss
}

\author{
(Denneth S. Henry, ${ }^{1,2}$ Mark Sayles, ${ }^{2,3}$ Ann E. Hickox, ${ }^{2}$ and ${ }^{\oplus}$ Michael G. Heinz ${ }^{2,3}$ \\ ${ }^{1}$ Department of Otolaryngology, University of Rochester, Rochester, New York 14642, ${ }^{2}$ Department of Speech, Language, and Hearing Sciences, Purdue \\ University, West Lafayette, Indiana 47907, and ${ }^{3}$ Weldon School of Biomedical Engineering, Purdue University, West Lafayette, Indiana 47907
}

\begin{abstract}
Speech intelligibility can vary dramatically between individuals with similar clinically defined severity of hearing loss based on the audiogram. These perceptual differences, despite equal audiometric-threshold elevation, are often assumed to reflect central-processing variations. Here, we compared peripheral-processing in auditory nerve (AN) fibers of male chinchillas between two prevalent hearing loss etiologies: metabolic hearing loss (MHL) and noise-induced hearing loss (NIHL). MHL results from age-related reduction of the endocochlear potential due to atrophy of the stria vascularis. MHL in the present study was induced using furosemide, which provides a validated model of age-related MHL in young animals by reversibly inhibiting the endocochlear potential. Effects of MHL on peripheral processing were assessed using Wiener-kernel (system identification) analyses of single AN fiber responses to broadband noise, for direct comparison to previously published AN responses from animals with NIHL. Wiener-kernel analyses show that even mild NIHL causes grossly abnormal coding of low-frequency stimulus components. In contrast, for MHL the same abnormal coding was only observed with moderate to severe loss. For equal sensitivity loss, coding impairment was substantially less severe with MHL than with NIHL, probably due to greater preservation of the tip-to-tail ratio of cochlear frequency tuning with MHL compared with NIHL rather than different intrinsic AN properties. Differences in peripheral neural coding between these two pathologies - the more severe of which, $\mathrm{NIHL}$, is preventable - likely contribute to individual speech perception differences. Our results underscore the need to minimize noise overexposure and for strategies to personalize diagnosis and treatment for individuals with sensorineural hearing loss.
\end{abstract}

Key words: auditory nerve; envelope; metabolic hearing loss; noise-induced hearing loss; temporal fine structure; Wiener-kernel analysis

\section{Significance Statement}

Differences in speech perception ability between individuals with similar clinically defined severity of hearing loss are often assumed to reflect central neural-processing differences. Here, we demonstrate for the first time that peripheral neural processing of complex sounds differs dramatically between the two most common etiologies of hearing loss. Greater processing impairment with noise-induced compared with an age-related (metabolic) hearing loss etiology may explain heightened speech perception difficulties in people overexposed to loud environments. These results highlight the need for public policies to prevent noiseinduced hearing loss, an entirely avoidable hearing loss etiology, and for personalized strategies to diagnose and treat sensorineural hearing loss.

\section{Introduction}

Individuals with the same clinical severity of hearing loss measured by the audiogram can show large differences in their ability

\footnotetext{
Received Jan. 7, 2019; revised May 8, 2019; accepted June 9, 2019.

Author contributions: K.S.H. and M.G.H. designed research; K.S.H., M.S., and A.E.H. performed research; K.S.H., M.S., A.E.H., and M.G.H. analyzed data; K.S.H. wrote the first draft of the paper; M.S., A.E.H., and M.G.H. edited the paper.

This work was supported by the National Institute on Deafness and other Communication Disorders (Grant F32-DC012236 to K.S.H. and Grant R01-DC009838 to M.G.H.). M.S. was supported by a UK-US Fulbright Commission scholarship award funded by Action on Hearing Loss.

The authors declare no competing financial interests.

Correspondence should be addressed to Michael G. Heinz at mheinz@purdue.edu or Kenneth S. Henry at kenneth_henry@urmc.rochester.edu.
}

to understand amplified speech (Dubno et al., 1982; Halpin and Rauch, 2009). Speech perception differences are commonly thought to reflect differences in cochlear innervation density (Bharadwaj et al., 2015), central processing, and cognition (Humes et al., 2012). Differences due to the etiology of sensorineural hearing loss (SNHL) have also been considered (Moore et al., 1999; Lopez-Poveda and Johannesen, 2012; Dubno et al., 2013). However, suprathreshold auditory nerve (AN) encoding

https://doi.org/10.1523/JNEUROSCI.0038-19.2019

Copyright $\odot 2019$ the authors 
of complex sounds has not been compared across different hearing loss etiologies.

Noise-induced hearing loss (NIHL) and metabolic hearing loss (MHL) are two common etiologies of SNHL, and often result in similar elevation of audiometric thresholds (Schuknecht and Gacek, 1993). In MHL, age-related deterioration of the stria vascularis reduces the electrochemical gradient (i.e., endocochlear potential) powering electromotility in outer hair cells and neurotransmitter release from inner hair cells (Mills et al., 2006; Schmiedt, 2010). With NIHL, threshold elevation arises differently due to scattered loss of and physical trauma to the delicate stereocilia of both hair cell types; more severe NIHL involves hair cell loss (Lurie et al., 1944; Liberman and Dodds, 1984; Sayles and Heinz, 2017). There are no previous studies on the effects of MHL on neural coding of complex sounds. It therefore remains unclear whether factors beyond the degree of threshold shift differ significantly between etiologies. Moreover, without direct comparison between NIHL and MHL, it is unknown whether this clinical subclassification is meaningful in terms of neural coding and could be potentially beneficial for individualized clinical rehabilitation.

The healthy cochlea decomposes complex sounds into an array of band-limited output signals, each representing the physical response of a frequency-tuned location within the cochlea. Each time domain output signal can be separated into a carrier signal (temporal fine structure; TFS) and envelope (ENV). TFS consists of oscillations near the characteristic frequency $(\mathrm{CF})$ of the cochlear location and the ENV describes slower variation in carrier signal amplitude over time. Both signals are encoded by AN fibers through spike timing (Joris and Yin, 1992). Fibers with CFs below 4-5 kHz encode both TFS and ENV, whereas higher-CF fibers encode primarily ENV (Recio-Spinoso et al., 2005).

Previous studies have used systems-identification techniques (e.g., Wiener-kernel analyses) to characterize AN fiber encoding of TFS and ENV with normal hearing and with NIHL (RecioSpinoso et al., 2005; Henry et al., 2016). Wiener-kernel analyses are based on responses to broadband noise, and thus quantify coding under suprathreshold conditions similar to those encountered in everyday life. Wiener-kernel analyses identify the stimulus-frequency components driving the fiber's TFS and ENV responses (Fig. 1A). Whereas control AN fibers encode TFS and ENV tonotopically (i.e., encoded frequency components are near CF; Fig. $1 B$, top), mild NIHL introduces pathological coding of below-CF TFS (Fig. 1B, middle). With moderate NIHL, ENV coding also becomes pathologically tuned to below-CF frequencies (Fig. 1B, bottom). These results demonstrate profound distortion of AN coding with even mild NIHL and that distortion progressively worsens to include ENV responses with increasing noise trauma.

The present study used Wiener-kernel analyses to quantify the effects of MHL on temporal coding in chinchilla AN fibers to allow direct comparison between MHL and NIHL effects. MHL was induced with furosemide, which provides a validated model of age-related MHL in young animals by reversibly inhibiting the endocochlear potential (Sewell, 1984; Ruggero and Rich, 1991; Rybak et al., 1992; Mills and Rubel, 1994; Schmiedt et al., 2002; Fitzgibbons and Gordon-Salant, 2010; Schmiedt, 2010). This approach allowed collection of neurophysiological responses associated with normal hearing and as a function of the degree of MHL in the same isolated fiber; that is, each neuron serves as its own control. Differences in suprathreshold AN coding of complex sounds after similar degrees of MHL and NIHL would help to explain individual differences in speech perception and pro-
A Wiener-kernel analyses
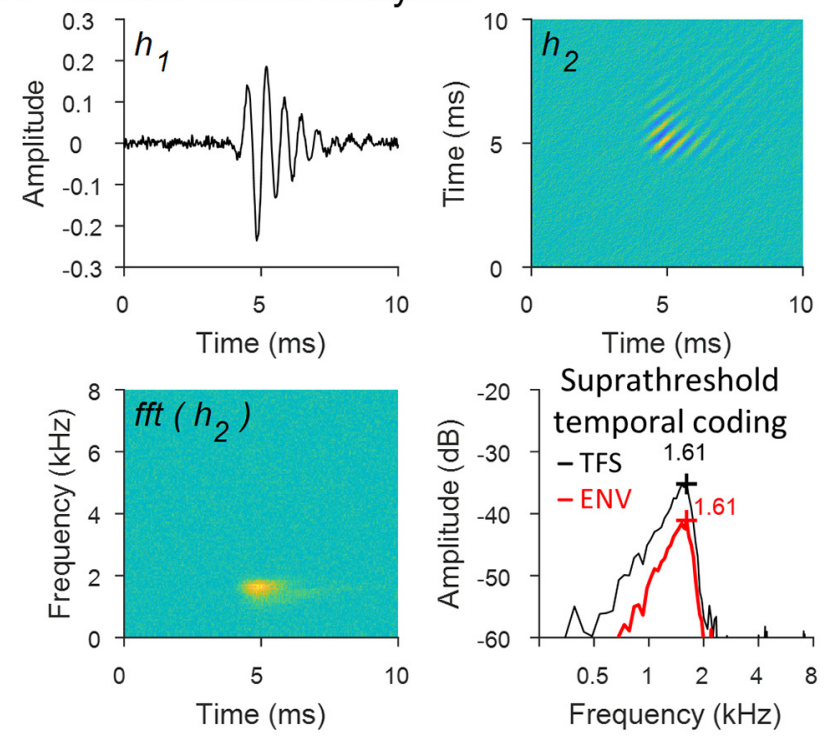

B Noise-induced hearing loss

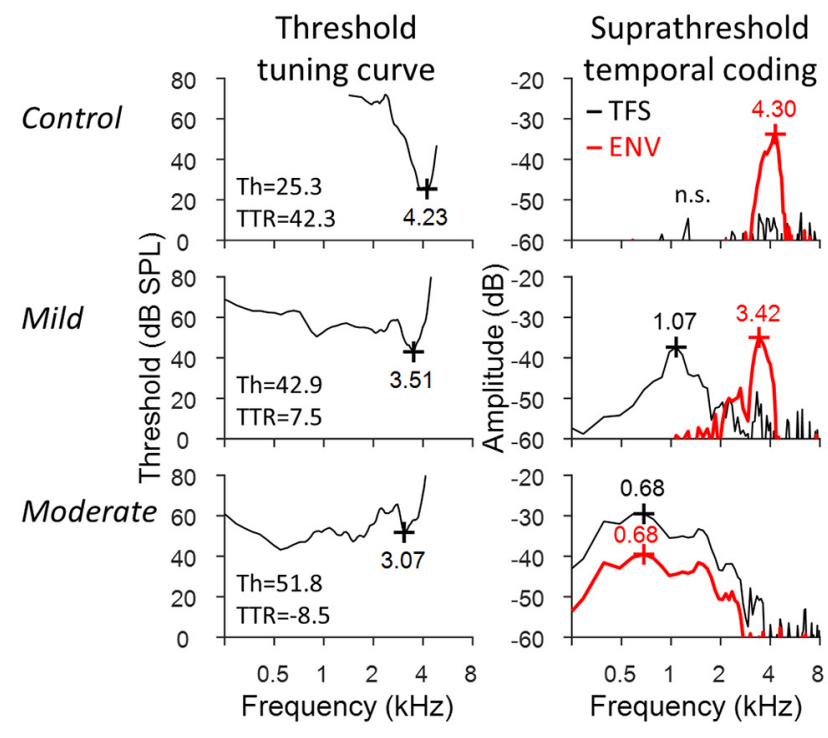

Figure 1. Neural system identification analyses of single-unit AN fiber responses reveal a significant loss of tonotopicity after NIHL for both TFS and ENV coding. $\boldsymbol{A}$, Wiener-kernel analyses of AN fiber responses to Gaussian noise used to study coding of TFS and ENV cues. The first-order Wiener kernel $\left(h_{1}\right)$ is the mean stimulus waveform before a spike. The second-order kernel $\left(h_{2}\right)$ is related to the spectrotemporal receptive field ( $\left.\mathrm{ftt}\left[h_{2}\right]\right)$ or mean stimulus spectrogram before a spike. $h_{1}$ identifies the frequency tuning of TFS coding. The first eigenvector of $h_{2}$ identifies the dominant frequency tuning of ENV coding; that is, the carrier-frequency band driving the fiber's time-varying response to ENV fluctuations. BF of suprathreshold tuning is given in kilohertz for both TFS (black) and ENV (red) coding. $\boldsymbol{B}$, Effects of NIHL on AN fiber coding of TFS and ENV. Pure tone tuning curves (left) were obtained from a control fiber (top) and two fibers with similar CF but different severity of NIHL. Hearing loss increases moving from top to bottom. CF of the threshold tuning curve is given in $\mathrm{kHz}$ near the tip. Threshold (Th) and TTR are given in $\mathrm{dB} S P L$ and $\mathrm{dB}$, respectively. The right column shows the frequency tuning of TFS coding and ENV coding obtained from Wiener-kernel analyses. BF of suprathreshold TFS/ENV coding is given in $\mathrm{kHz}$ near the peak of each curve (n.s. indicates nonsignificant coding amplitude $<3$ SDs above the noise floor). The control fiber encodes only ENV structure near (F. Mild NIHL introduces pathological coding of low-frequency TFS. Moderate NIHL shifts coding of both TFS and ENV to low frequency (data from Henry et al., 2016). 
vide physiological support for translational efforts to dissect clinical subcategories of SNHL.

\section{Materials and Methods}

Animal procedures. All procedures were performed in male chinchillas $(n=7)$ and approved by the Purdue University Animal Care and Use Committee. Anesthesia was induced with xylazine ( $1-2 \mathrm{mg} / \mathrm{kg}$, s.c.) followed several minutes later by ketamine $(50-65 \mathrm{mg} / \mathrm{kg}$, i.p. $)$. Atropine $(0.05 \mathrm{mg} / \mathrm{kg}$, i.m.) was given to control mucous secretions and eye ointment was applied. Anesthesia was maintained with bolus injections of sodium pentobarbital ( $\sim 15 \mathrm{mg} / \mathrm{kg}$, intravenous) or ketamine $(20-40 \mathrm{mg} / \mathrm{kg})$ with diazepam $(1-2 \mathrm{mg} / \mathrm{kg}, \mathrm{i} . \mathrm{m}$.$) , given once per \sim 2$ $\mathrm{h}$ period for the duration of experimental procedures (typically $10-12$ h). Physiological saline (1-2 $\mathrm{ml} / 2$ h, i.v.) and lactated Ringer's solution $(20-30 \mathrm{ml} / 24 \mathrm{~h}$, s.c.) were also given and a tracheotomy was performed to maintain a low-resistance airway. Anesthetized animals were positioned with a stereotaxic device, and body temperature was maintained at $37^{\circ} \mathrm{C}$ using a feedback-controlled heating pad (Harvard Apparatus 50-7220F).

The skin and muscles overlying the skull were reflected to expose the bony ear canals and bullae. Both ear canals were dissected to allow insertion of hollow ear bars close to the tympanic membrane. The AN bundle was exposed at its exit from the internal acoustic meatus via a posteriorfossa craniotomy and aspiration cerebellotomy. Acoustic stimuli were presented monaurally through an ear bar with a dynamic loudspeaker (Beyerdynamic DT48 or Etymotic ER2) and calibrated using a probe microphone placed within a few $\mathrm{mm}$ of the tympanic membrane (Etymotic ER7C). Neurophysiological recordings were made using 10-50 $\mathrm{M} \Omega$ impedance glass micropipettes advanced into the AN using a hydraulic microdrive. Recordings were amplified (Dagan 2400A) and band-pass filtered from $0.03-6 \mathrm{kHz}$ (Krohn-Hite 3550). Spikes were identified using a time-amplitude window discriminator (BAK Electronics) and stored as spike times with $10 \mu$ s resolution.

Single fibers were isolated by listening for spikes on a monitor speaker while advancing the electrode through the AN during repeated stimulation with broadband noise. When a fiber was encountered, a tuning curve was recorded using an automated procedure that tracked, as a function of stimulus frequency, the minimum SPL of a $50 \mathrm{~ms}$ tone required to evoke at least 1 more spike than a subsequent $50 \mathrm{~ms}$ silent period (Chintanpalli and Heinz, 2007). Next, a rate level function was recorded with broadband Gaussian noise stimuli to determine the noise threshold. Noise stimuli were $2.5 \mathrm{~s}$ in duration with a bandwidth of $16.5 \mathrm{kHz}$ and $1 \mathrm{~s}$ silent interval between stimuli. Finally, 10 independent Gaussian noise stimuli were presented repeatedly for 3-5 min at $10-20 \mathrm{~dB}$ above the noise threshold until $\sim 5000-10,000$ driven spikes were recorded. These responses were used for the Wiener-kernel analyses.

After collection of control data, furosemide was administered $(25 \mathrm{mg} /$ $\mathrm{kg}$, i.v.) to induce MHL. Pure tone tuning curves (2 $\mathrm{min}$ ), noise rate level functions ( $1 \mathrm{~min}$ ), and suprathreshold noise responses (3-5 $\mathrm{min}$ ) were recorded repeatedly in this order until the fiber was lost (40-60 min in most cases). The sound level used for the suprathreshold noise response was selected based on the preceding noise rate level function to be 10-20 $\mathrm{dB}$ above threshold, and varied from 35 to $100 \mathrm{~dB}$ SPL depending on the degree of MHL. Data from noise-exposed and additional control fibers were collected in a previous study (Henry et al., 2016) using the same methods. Intravenous injections of physiological saline during AN recordings had no impact on neural response properties in noise-exposed and control fibers.

Tuning curve $\mathrm{CF}$ was identified as the frequency of best sensitivity (i.e., threshold) to pure tone stimuli. In rare cases where tuning curves lacked a clear tip (MHL: $0 \%$ of fibers; NIHL: $4.7 \%$ of fibers), CF was estimated using the breakpoint in the high-frequency slope of the tuning curve (Liberman, 1984). Tip-to-tail ratio (TTR) was calculated as the threshold 1.5 octaves below CF minus the threshold at CF (Temchin et al., 2008). Normalized TTR was calculated by subtracting the mean value in normal-hearing control fibers of the same CF. Mean TTR in chinchilla AN fibers increases from $27.5 \mathrm{~dB}$ for CFs below $3 \mathrm{kHz}$ to $48 \mathrm{~dB}$ above 4 $\mathrm{kHz}$ (Temchin et al., 2008). In addition to CF, best frequency (BF, the frequency of maximal response amplitude) was used to characterize suprathreshold tuning from broadband-noise responses.

Wiener-kernel analyses of neural responses. The basic Wiener-kernel computations have been described previously (Eggermont et al., 1983; van Dijk et al., 1994; Lewis et al., 2002; Recio-Spinoso et al., 2005; van Drongelen, 2010). Briefly, $h_{1}$ and $h_{2}$ were calculated based on first- and second-order cross-correlations, respectively, between the noise stimulus waveform $x(t)$ and response train of $n=5000-10,000$ driven spikes. Only spikes occurring $>20 \mathrm{~ms}$ after stimulus onset and before stimulus offset were included in the correlations, which were calculated with a sampling period of $0.02 \mathrm{~ms}$ and maximum time lag $\tau$ of $10.2 \mathrm{~ms}$ (512 points) or $20.4 \mathrm{~ms}$ (1024 points; for $\mathrm{CFs}<3 \mathrm{kHz}$ ). $h_{1}$ was calculated as

$\frac{1}{\sqrt{A}} R_{1}(\tau)$, where $A$ is the mean power of the noise stimulus and $R_{1}(\tau)=\frac{1}{N} \sum_{i=1}^{N} x\left(t_{i}-\tau\right)$ is the reverse correlation function and $t_{\mathrm{i}}$ the time of the $i^{\text {th }}$ spike expressed relative to stimulus onset. So computed, $h_{1}$ is a time waveform that reflects the normalized mean pattern of TFS preceding a spike (Fig. $1 A$, top left). The BF of TFS coding was calculated as the frequency of the dominant component in the Fourier transform of $h_{1}$ (Fig. $1 A$, bottom right, black). The amplitude of TFS coding was calculated as the peak of the Hilbert envelope of $h_{1}$. The strength of below-CF TFS coding was calculated as the peak of the Hilbert envelope of a low-pass filtered $h_{1}$. Low-pass filtering was accomplished in the frequency domain by calculating the Fourier transform of $h_{1}$, multiplying components with frequencies greater than $\mathrm{CF} / 2$ by 0 , and calculating the inverse Fourier transform.

$$
h_{2}\left(\tau_{1}, \tau_{2}\right) \text { was calculated as } \frac{1}{2 A}\left[R_{2}\left(\tau_{1}, \tau_{2}\right)-\phi_{x x}\left(\tau_{2}-\tau_{1}\right)\right] \text {, where } \tau_{1}
$$
and $\tau_{2}$ are time lags, $R_{2}\left(\tau_{1}, \tau_{2}\right)=\frac{1}{N} \Sigma_{i=1}^{N} x\left(t_{i}-\tau_{1}\right) x\left(t_{i}-\tau_{2}\right)$ is the second-order reverse-correlation function, and $\phi_{\mathrm{xx}}(\tau)$ is the autocorrelation function of the stimulus. So computed, $h_{2}$ is a surface that captures nonlinear interaction in the response to two impulses (Fig. 1A, top right). The 1D Fourier transform of $h_{2}$ provides a representation of the spectrotemporal receptive field or mean stimulus spectrogram occurring before a spike (Lewis and van Dijk, 2004) (Fig. $1 A$, bottom left). In practice, $h_{2}$ can contain bands running parallel to the diagonal, reflecting phase locking to ENV, and perpendicular to the diagonal, reflecting nonlinearity in the phase-locked response to TFS. When both occur in a single fiber, a checkerboard pattern emerges. The spectral energy of the ENV-based component of $h_{2}$ is restricted to the second and fourth quadrants of its 2-dimensional Fourier transform, whereas the spectral energy of the TFS-based component is restricted to the first and third quadrants (Recio-Spinoso et al., 2005). We multiplied the contents of the first and third quadrants by zero to yield a filtered second-order kernel based primarily on ENV coding (Henry et al., 2016). We used eigenvector decomposition to quantify the properties of $h_{2}$ as in previous studies (Lewis et al., 2002; Recio-Spinoso et al., 2005). Eigenvector decomposition of $h_{2}$ yielded one or sometimes two pairs of eigenvectors (in quadrature phase) with significant amplitude ( $\geq 3$ SDs above the noise floor). The BF of ENV coding was determined from the Fourier transform of these eigenvectors (Fig. 1A, bottom right, red). The amplitude of ENV coding was calculated as the peak of the Hilbert envelope of the first $h_{2}$ eigenvector. The strength of below-CF ENV coding was calculated as the peak of the Hilbert envelope of a low-pass filtered first eigenvector. Filtering was performed in the frequency domain as described above for TFS with a cutoff frequency of $\mathrm{CF} / 2$.

Experimental design and statistical analysis. Coding deficits were analyzed in R (version 3.4.1) using linear mixed-effects models (Bates et al., 2015). Dependent variables were the BF shift of coding, calculated as the frequency difference in octaves between $\mathrm{BF}$ and the tuning curve $\mathrm{CF}$, and below-CF coding amplitude. Fixed effects included hearing loss type (MHL vs NIHL), coding mechanism (TFS vs ENV), and CF threshold. A unique identifier for each AN fiber was used as the random effect. Interactions were included between fixed effects and dropped when not significant $(p<0.05)$ in order of decreasing $p$-value. Degrees of freedom for $F$ tests and pairwise comparisons of least-squared means were calculated 


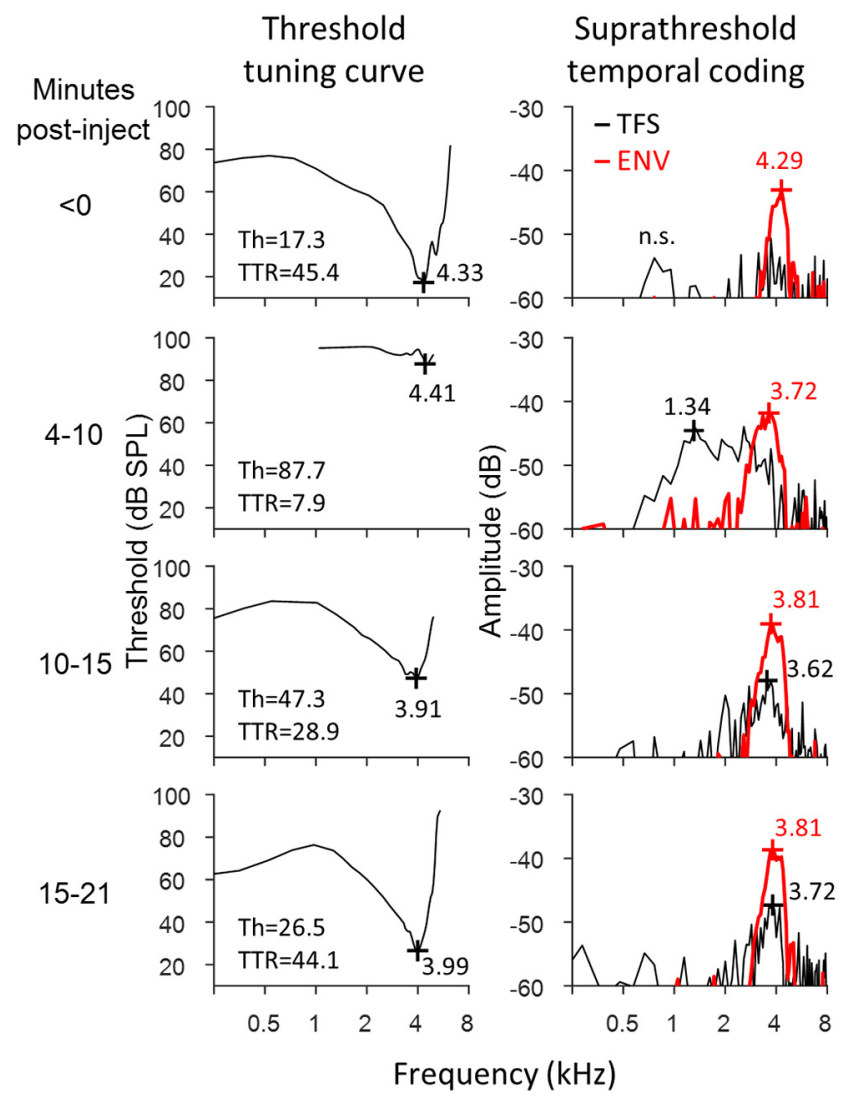

Figure 2. Variation over time after furosemide injection in threshold tuning curves (left) and suprathreshold temporal coding (right) of a representative AN fiber. TFS and ENV coding remain tuned near CF during moderate MHL. Severe MHL, during which CF thresholds exceed $70 \mathrm{dBSPL}$, introduces pathological coding of low-frequency TFS. CF, threshold, and TTR are given for tuning curves and BF for suprathreshold TFS/ENV coding as in Figure 1. Spontaneous rate decreased from 76.6 to 2.2 spikes/s after furosemide injection before increasing to 10.6 spikes/s after 21 min of recovery. Lower spontaneous rate near the end of the recovery period in this fiber (bottom row) may explain the slight enhancement of (F-tuned TFS/ENV coding compared with control data. n.s., not significant.

based on the Satterthwaite approximation (Bates et al., 2015). Visual inspection of model results showed that residuals were normally distributed after log transformation of below-CF coding amplitude. No transformation of BF shift was required.

\section{Results}

Effects of MHL on AN coding of broadband sounds were studied in seven animals and compared with the effects of NIHL reported in a previous study (Henry et al., 2016). System identification (Wiener-kernel) analyses were applied to spike train responses to broadband noise $(n=75)$ obtained from 28 fibers, including 14 fibers tested across multiple levels of furosemide-induced MHL.

Control responses, recorded immediately before furosemide injection, showed typical tonotopic coding of TFS and $\mathrm{ENV}$, consistent with previous results in normal-hearing animals (Recio-Spinoso et al., 2005). For AN fibers with CFs below $4-5 \mathrm{kHz}$, the BFs of suprathreshold TFS and ENV coding were both closely tuned to the threshold CF of the pure tone tuning curve (a traditional index of cochlear location) (Liberman, 1982). In fibers of higher CF, the BF of ENV coding closely matched CF, while TFS coding was weak or not significant due to a decline in TFS response amplitude with increasing CF (Fig. 2, top row) that was consistent with the roll-off in phase locking for pure tone responses (Johnson, 1980). AN responses associated with "normal" thresholds ( $\leq 30 \mathrm{~dB}$ SPL) showed no substantial temporal (TFS or ENV) coding of stimulus components $>1$ octave below CF (Fig. $3 C, D$; blue triangles: furosemide experiments, $n=28$; black crosses: control experiments, $n=37$; Henry et al., 2016).

Tuning curve thresholds at CF increased by $30-70 \mathrm{~dB}$ within 2 min of furosemide injection, followed by a gradual recovery of sensitivity over 30-120 min (Fig. 2, left column; Fig. 4A). Tonotopic coding of TFS and ENV were largely maintained with mild to moderate MHL, during which CF thresholds ranged from 30 to $65 \mathrm{~dB}$ SPL. Figure 4 shows representative longitudinal data from a fiber across varying degrees of mild to moderate MHL. Peak threshold elevation was associated with minor downward BF shifts in suprathreshold TFS/ENV coding (Fig. 4B), increased tuning bandwidth, and slightly stronger encoding of below-CF stimulus components (Fig. 4C). To compare response properties across fiber populations, BF shifts of suprathreshold TFS and ENV coding were calculated relative to CF on an octave scale. As in control fibers, the BF of ENV coding in the mild to moderate MHL fiber population remained tuned near CF (typically within 0.5 octaves; Fig. $3 B$; blue triangles, $n=28$ ), while TFS coding remained either tuned near CF (Fig. 2, 10-21 min post injection; see also Fig. 4 ) or low in amplitude (for higher CFs, as in control fibers; Fig. $3 A$; blue triangles, $n=28$ ). Mild to moderate MHL was associated with a modest increase in the below-CF amplitude of TFS coding, but minimal change in ENV coding (Fig. 3C,D).

Moderate to severe MHL, during which thresholds exceeded $65 \mathrm{~dB}$ SPL, was commonly associated with a substantial downward shift in the BF of TFS coding below the tuning curve CF and a concomitant increase in the amplitude of abnormal below-CF TFS coding (Fig. $3 A, C$; blue triangles, $n=17$ ). Indeed, coding of low-frequency TFS occurred even in high-CF fibers that showed no TFS coding before furosemide injection (Fig. 2; 4-10 min after injection). These patterns likely result from furosemideinduced changes in cochlear frequency tuning and nonlinear suppression (Ruggero and Rich, 1991; Ruggero et al., 1992), including lower TTR, as further considered below. Some cases of moderate to severe MHL also caused downward shifts in the BF of ENV coding and an increase in the amplitude of below-CF ENV coding (Fig. 3B,D). Tonotopic coding deficits were generally smaller for ENV than for TFS for the same severity of threshold elevation.

Broadband noise responses from previous NIHL experiments (Henry et al., 2016) were reanalyzed to allow direct comparison of AN coding between MHL and NIHL as a function of degree of hearing loss. As with MHL, coding deficits with NIHL included downward BF shifts in TFS and ENV coding and related increases in the amplitude of below-CF coding (Fig. 3; red circles, $n=62$ ). As with MHL, tonotopic coding deficits were greater for TFS than for ENV coding. NIHL produced substantially larger tonotopic coding deficits than MHL for equal impaired CF threshold. Indeed, the substantial tonotopic deficits in TFS coding observed with mild NIHL (thresholds $\geq 30-40 \mathrm{~dB}$ SPL) were observed only in moderate to severe instances of MHL (thresholds $\geq 60-70 \mathrm{~dB}$ SPL).

Mixed-model analyses of the subset of responses associated with mild to moderate loss (CF thresholds between 30 and $65 \mathrm{~dB}$ SPL) confirmed greater tonotopic coding deficits with NIHL $(n=57)$ than with MHL $(n=28)$. The analysis of BF shifts showed significant effects of hearing loss type (MHL vs NIHL; $\left.F_{(1,2.4)}=58.11, p<0.0001\right)$, coding mechanism (TFS vs ENV; 


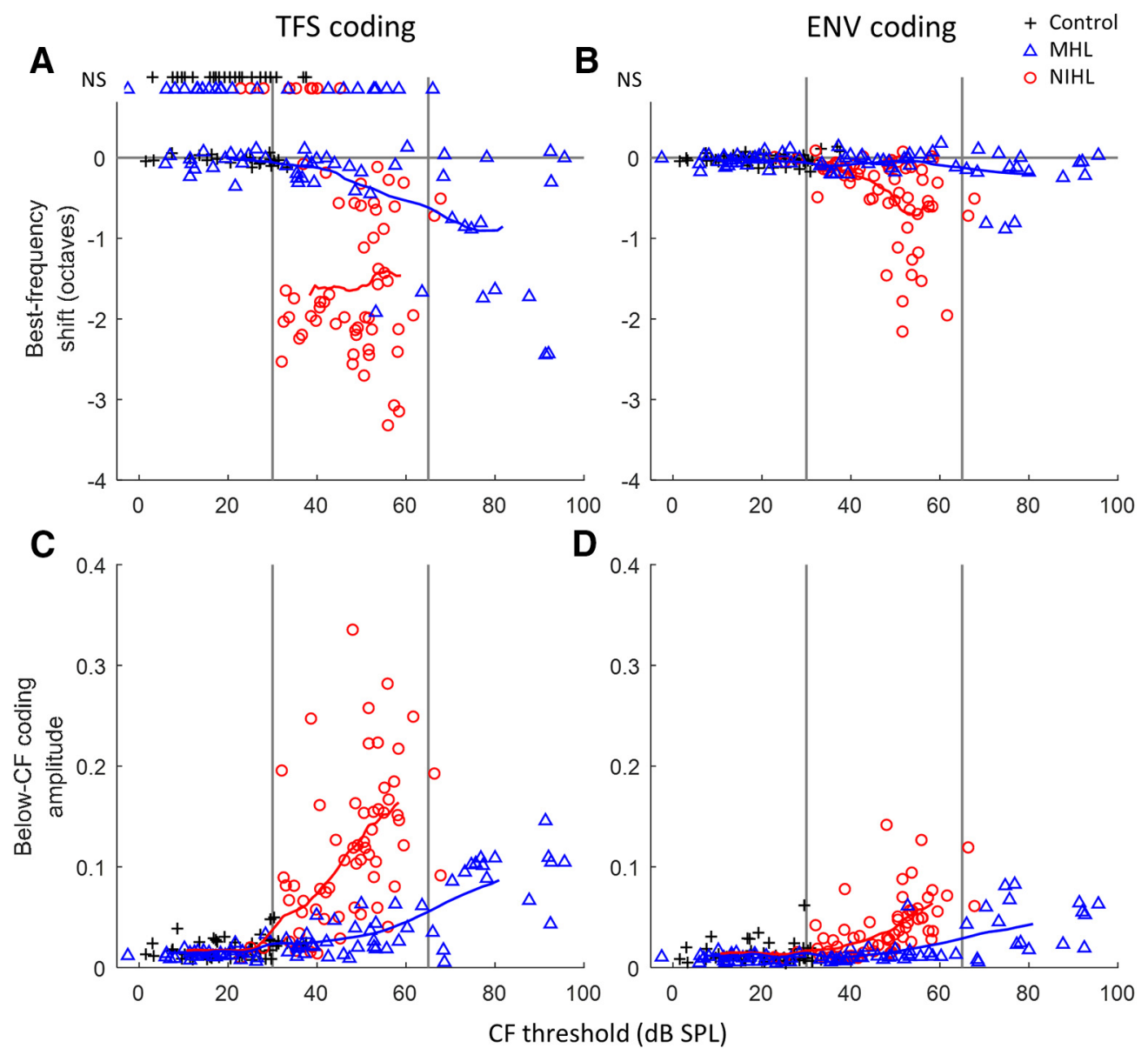

Figure 3. TFS and ENV coding deficits are greater with NIHL than with MHL for the same impaired CF threshold. Coding metrics include BF shifts in TFS coding ( $A$ ), BF shifts in ENV coding ( $\boldsymbol{B})$, below-CF amplitude of TFS coding $(\boldsymbol{C})$, and below-CF amplitude of ENV coding (D). Metrics are compared between MHL, NIHL, and control fibers (see key) as a function of the tuning curve threshold at $C F$. BF shift indicates the frequency difference in octaves between the BF of coding and the tuning curve CF. Below-CF amplitude indicates the strength of coding for stimulus components one or more octaves below CF. Note that for both metrics of temporal coding (for both TFS and ENV), significant degradations are observed after NIHL for CF thresholds as low as $30 \mathrm{~dB}$ SPL, whereas similar degrees of degradations are not seen after MHL until $\sim 65 \mathrm{~dB} \mathrm{SPL}$ CF thresholds. Trend lines show the moving average (16 points) for MHL and NIHL. NS, Not significant. CFs range from 1.24 to $10.5 \mathrm{kHz}$.

$\left.F_{(1,110.1)}=56.28, p<0.0001\right)$, and the hearing loss type by coding mechanism interaction $\left(F_{(1,108.8)}=18.12, p<0.0001\right)$. The effect of CF threshold over this limited range was non-significant $\left(F_{(1,49.6)}=3.25, p=0.078\right)$. Pairwise differences in least-squared means $( \pm \mathrm{SE})$ showed that, compared with MHL, NIHL caused larger downward shifts in TFS-coding BF $(-1.307 \pm 0.163$ octaves; $t_{(55.7)}=-8.04, p<0.0001$; Fig. $3 A$ ) and, to a lesser extent, ENV coding BF $\left(-0.399 \pm 0.146\right.$ octaves; $t_{(43.9)}=-2.73, p=$ 0.009 ; Fig. $3 B$ ). The analysis of log-transformed below-CF coding amplitude showed significant effects of hearing loss type $\left(F_{(1,49.2)}=48.90, p<0.0001\right)$, coding mechanism $\left(F_{(1,98.4)}=\right.$ $212.21, p<0.0001)$, CF threshold $\left(F_{(1,139.5)}=42.15, p<0.0001\right)$, and the hearing loss type by coding mechanism interaction $\left(F_{(1,98.4)}=5.35, p=0.023\right)$. Compared with MHL, NIHL induced stronger below-CF coding of TFS (11.19 $\pm 1.52 \mathrm{~dB}$; $t_{(68.3)}=7.35, p<0.0001$; Fig. $\left.3 C\right)$ and $\operatorname{ENV~}(8.50 \pm 1.52 \mathrm{~dB}$; $t_{(68.3)}=5.58, p<0.0001$; Fig. $\left.3 D\right)$.

These substantial tonotopic coding differences across hearing loss types must result from differences in the effects of NIHL and MHL beyond what is captured simply by CF threshold. The tuning curve tip-to-tail ratio (TTR; i.e., threshold difference between the below-CF tail region and the CF tip) provides a different tuning index that depends on various factors associated with co- chlear hearing loss that may differ between NIHL and MHL (Liberman and Dodds, 1984). TTR was reduced as CF threshold increased for both NIHL and MHL (Fig. 5). The reduction in TTR was less with MHL than with NIHL for comparable CF thresholds (Fig. 5), consistent with hypersensitive tails that occur after NIHL (Liberman and Dodds, 1984) but not after MHL (Sewell, 1984). The temporal coding deficits were much more similar between NIHL and MHL when plotted as a function of TTR reduction (Fig. 6) than when plotted as a function of CF threshold (Fig. 3). These findings suggest that the factors underlying TTR, which are putatively related to differences in cochlear micromechanics, may largely account for the variation in deficits observed for broadband sound encoding, independent of hearing loss type (MHL vs NIHL); however, some evidence for greater effects with NIHL remains for ENV coding, suggesting that whereas TTR may be the primary factor, other factors may also contribute.

We used a simple AN model to help address whether changes in the TTR of cochlear frequency tuning can account for the observed coding deficits without requiring an explicit deficit in the ability of AN fibers to phase lock. The first stage of the model was a linear band-pass filter with tip and tail components (500point FIR), to simulate the mechanical response of the $4 \mathrm{kHz}$ 

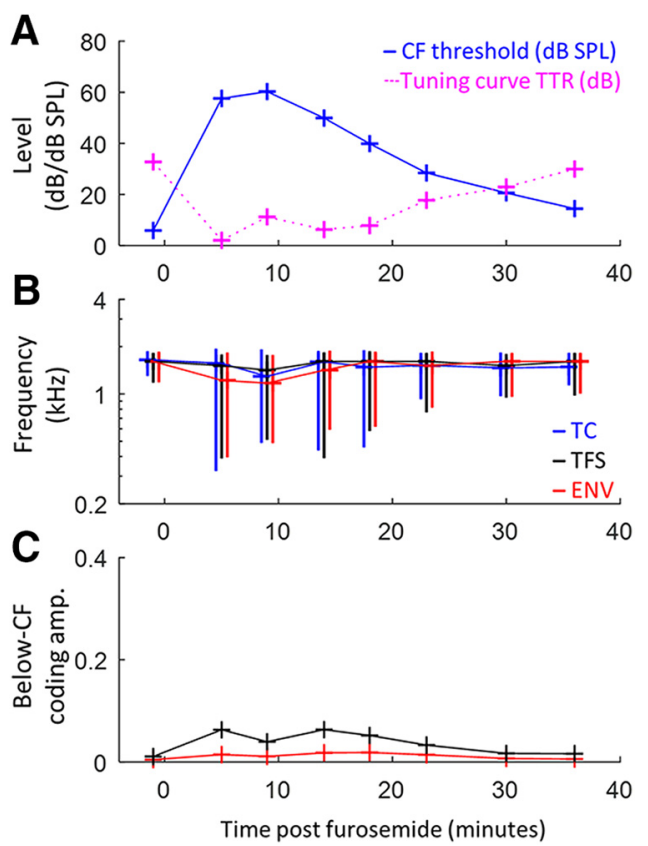

Figure 4. Dynamic variation in suprathreshold temporal coding over varying degrees of mild to moderate MHL in a representative AN fiber. Change over time after furosemide injection in tuning curve threshold and TTR $(A), B F$ s of suprathreshold TFS/ENV coding and tuning curve $C F(B)$, and below-CF amplitude of TFS/ENV coding $(\boldsymbol{C})$. Vertical lines in $\boldsymbol{B}$ indicate the $10 \mathrm{~dB}$ bandwidth of frequency tuning. Limits of the $y$-axis in C match Figure 3, C and $D$, for direct comparison of the smaller MHL effects compared with NIHL effects. MHL peaked from 5-10 min after furosemide injection and recovered almost completely within $35 \mathrm{~min}$. Tonotopic coding of TFS and ENV was generally well preserved during mild to moderate MHL.

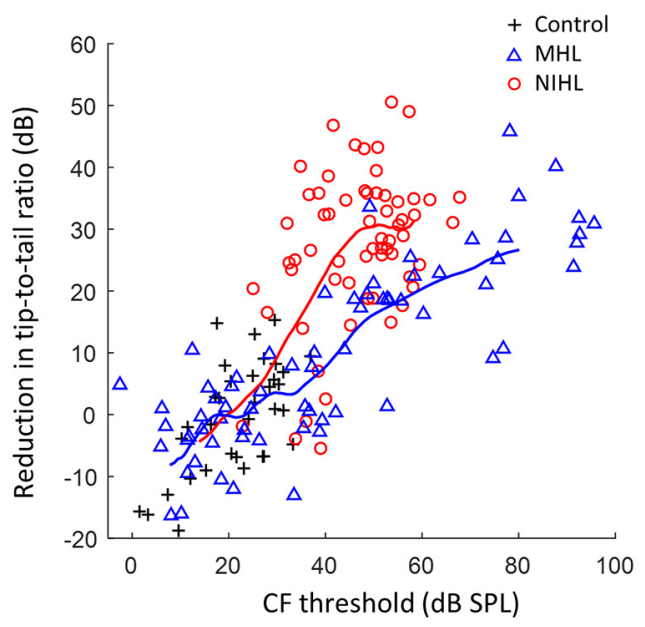

Figure 5. Reduction in tuning curve TTR is greater with NIHL than with MHL for the same impaired CF threshold. TTR was quantified 1.5 octaves below $C F$ and normalized for the CF dependence in normal-hearing animals (Temchin et al., 2008). Trend lines show the moving average (16 points) for MHL and NIHL. CFs range from 1.24 to $10.5 \mathrm{kHz}$.

location within the cochlea (Fig. 7A; tuning curves show the inverted frequency response of the filter). Output from the filter was half-wave rectified and low-pass filtered to capture the low-pass nature of inner hair cell responses and AN phase locking (Heinz et al., 2001). Suprathreshold coding of TFS and ENV were quantified using Wiener-kernel analyses of the model response to broadband noise (Fig. $7 B$ ), as applied above to physiological AN responses. The normal-hearing model fiber with TTR of $40 \mathrm{~dB}$ showed tonotopic encoding of TFS and
ENV, as expected. Decreasing TTR to 15 and then $5 \mathrm{~dB}$ increased suprathreshold encoding of low-frequency TFS and, to a lesser extent, of ENV structure. These results are consistent with more physiologically specific computational modeling (Heinz and Henry, 2013), and suggest that differences in the TTR of cochlear frequency tuning underlie the observed tonotopic coding deficits for broadband sound, including greater impairment of TFS coding than ENV coding and greater impairment with NIHL than MHL.

\section{Discussion}

Similar to NIHL, MHL can induce pathological changes in tonotopic coding of temporal information. Moderate to severe MHL induced pathological AN coding of low-frequency TFS well below CF during stimulation with broadband noise. Deficits in ENV coding also occurred, but were smaller than TFS coding deficits. For both MHL and NIHL, temporal coding degradation increased with the degree of hearing loss, but with greater coding impairment for NIHL than for MHL when compared at equal impaired threshold (Fig. 3). Differences in the degree of temporal-coding deficits between MHL and NIHL were largely unified when considered in terms of tuning curve TTR (Fig. 6), suggesting that deficits result from changes in cochlear frequency tuning rather than intrinsic temporal properties of AN fibers. Furthermore, these results suggest that factors beyond the degree of hearing loss are important for dissecting the clinical category of SNHL when considering suprathreshold processing of complex sounds.

Pathological coding of low-frequency TFS with moderate to severe MHL is likely a consequence of significantly reduced OHC gain resulting from a greatly diminished endocochlear potential (Ruggero and Rich, 1991). This reduction in "battery power" for cochlear amplification decreases sensitivity to frequency components near CF (the "tip") and ultimately decreases the ratio of tip sensitivity to below-CF "tail" sensitivity. Diminished OHC function (lower TTR) decreases the ability of AN fibers to reject low-frequency input, consistent with the abnormal response to low-frequency TFS and the observed relationships between coding defects and CF-normalized TTR (Fig. 6). Healthy OHCs enable normal fibers with high TTRs to encode temporal structure in the on-CF band rather than the tail region of the stimulus (i.e., to maintain tonotopicity).

Translationally, the most important result was that deficits in temporal coding arising from a certain degree of hearing loss were more severe when they arose from NIHL rather than MHL. This result suggests that not all SNHL etiologies affect AN coding of complex sounds identically. Pathological coding of low-frequency TFS, for instance, required CF thresholds only in excess of $30-40 \mathrm{~dB}$ SPL with NIHL versus $60-70 \mathrm{~dB}$ SPL with MHL (Fig. 3). Moreover, loss of ENV coding near $\mathrm{CF}$, as indicated by BF shifts greater than one octave, was observed for NIHL with moderate threshold elevation $(>50$ dB SPL), but was never observed with MHL even when CF thresholds exceeded $90 \mathrm{~dB}$ SPL.

Greater coding deficits with NIHL were closely associated with greater reductions in TTR for NIHL than for MHL with equal CF thresholds (Figs. 5, 6). Both changes in cochlear function (i.e., suprathreshold coding of complex sounds and threshold tuning curves) likely arise from the same mechanisms. As with MHL, nonlinear $\mathrm{OHC}$ gain is reduced (thus elevating tips) with NIHL, but perhaps to a greater degree based on previous studies showing greater preservation of two-tone suppression (Schmiedt et al., 1990) and otoacoustic emissions (Mills et al., 

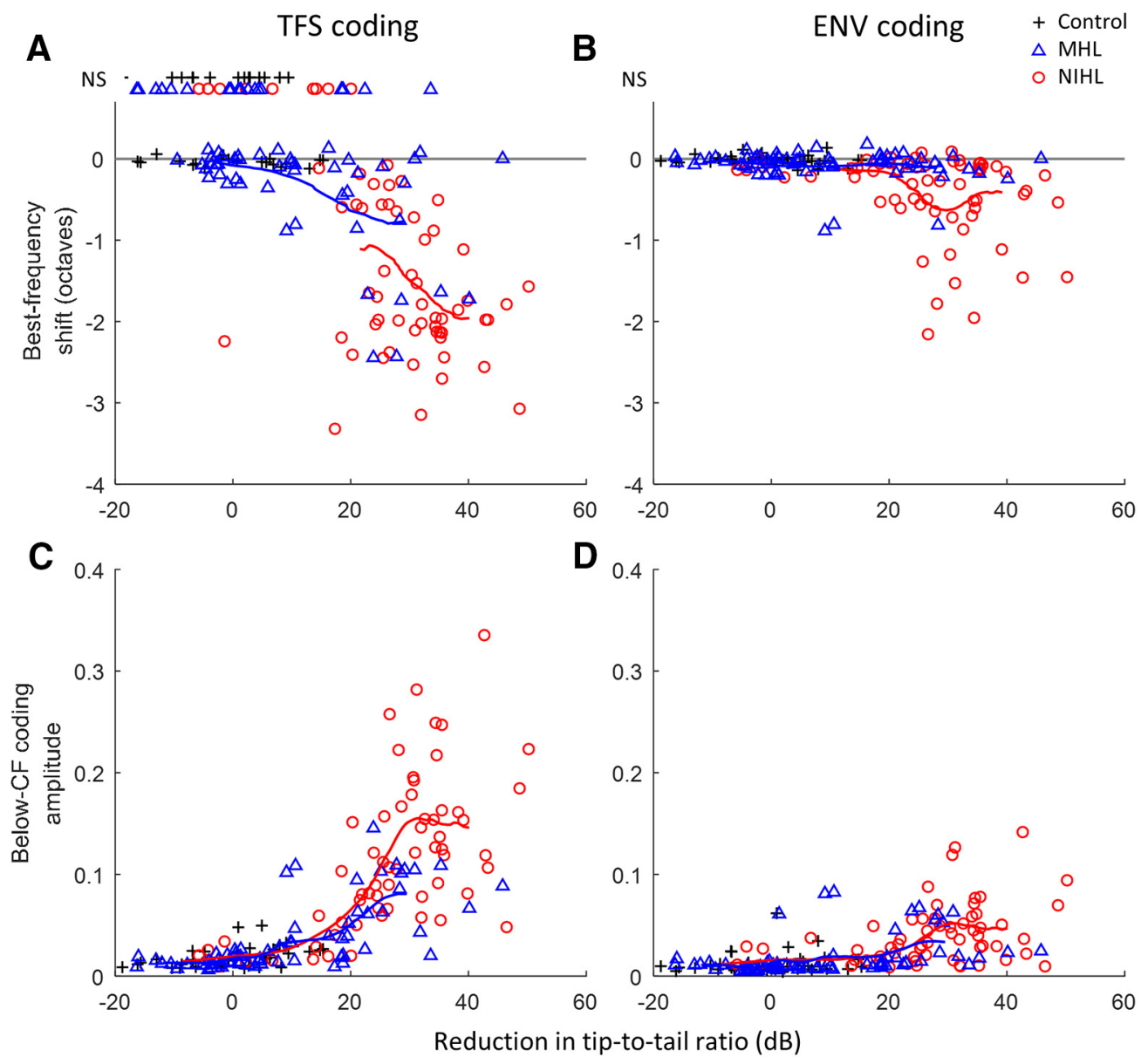

D

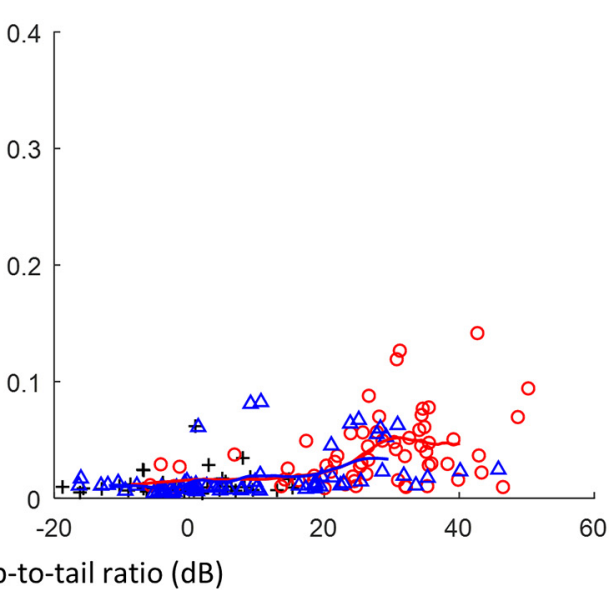

Figure 6. Greater TFS coding deficits for NIHL than for MHL are accounted for when compared at equal reduction in the TTR. However, ENV coding remains somewhat worse for NIHL than for MHL even when TTR is considered. Thus, reduction in TTR appears to be the primary (but not only) factor, rather than (F threshold, in explaining the degradations in TFS and ENV tonotopic coding. Coding metrics are the same as in Figures 3 and 4: BF shifts in TFS $(\boldsymbol{A})$ and ENV coding $(\boldsymbol{B})$, and below-CF amplitude of TFS ( $\boldsymbol{C}$ ) and ENV coding (D). Trend lines show the moving average (16 points) for MHL and NIHL. NS, not significant. CFs range from 1.24 to $10.5 \mathrm{kHz}$.

\section{A Model threshold tuning curve}
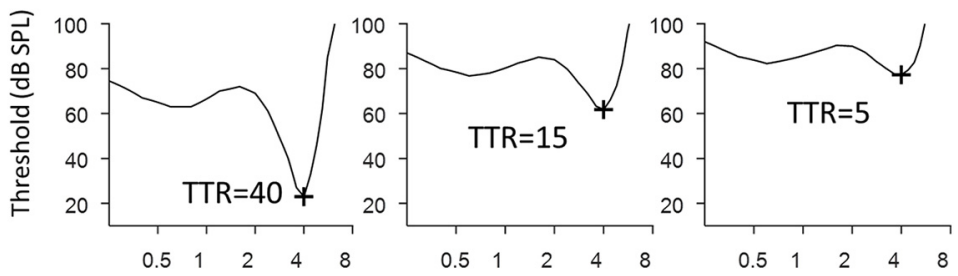

B Model suprathreshold coding

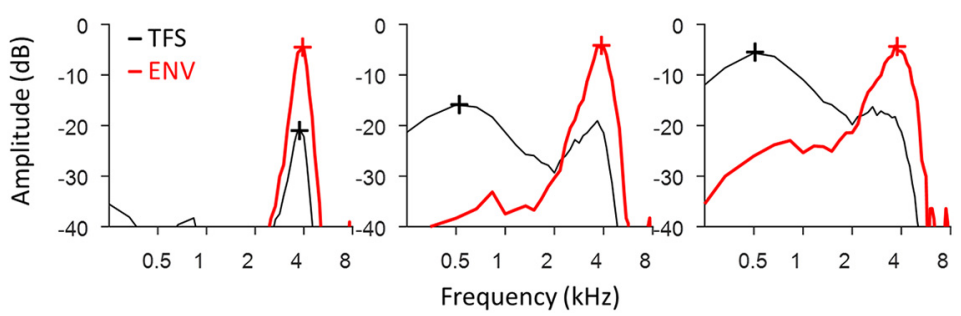

Figure 7. Predicted relationship between TTR of cochlear frequency tuning and suprathreshold AN encoding of broadband sound. $\boldsymbol{A}$, The model consists of a band-pass filter with tip and tail components, followed by half-wave rectification and low-pass filtering to capture the roll-off of AN phase locking with increasing frequency. $\boldsymbol{B}$, Suprathreshold coding of TFS and ENV, quantified using Wiener-kernel analyses of model responses to broadband noise. Lower TTR of cochlear frequency tuning is associated with increased coding of low-frequency stimulus component and greater coding deficits for TFS than ENV, as observed physiologically. Note that the roll-off of AN phase locking remained unchanged in the model, suggesting that effects of NIHL/MHL on AN temporal coding result from changes in the TTR of cochlear frequency tuning, rather than an explicit deficit in the fundamental phase-locking ability of AN fibers.
1993; He and Schmiedt, 1996; Schmiedt et al., 2002) with MHL compared with NIHL. Uniquely contributing to NIHL is the phenomenon of hypersensitive tails, where below-CF thresholds can be reduced (rather than elevated) after selective outer hair cell damage (Liberman and Dodds, 1984). Mechanisms underlying tail hypersensitivity are not well understood, but may relate to reduced organ of Corti stiffness associated with loss of the physical coupling (via the outer hair cells) between the reticular lamina and tectorial membrane. Note that reduced stiffness generally decreases the resonant frequency of a passive physical system. In summary, the combined effects of greater OHC gain reduction and tail hypersensitivity mechanisms appear to heighten the effect of NIHL on suprathreshold coding of complex sounds compared with MHL, even in cases of mild to moderate hearing loss.

Due to threshold elevation, higher levels were used for NIHL (47-93 dB SPL) 
and for MHL (35-100 dB SPL) compared with control experiments (32-66 dB SPL), raising the question of how these groups would compare at equal sound level. Although not addressed directly in the present study, previous Wiener-kernel analyses of normal-hearing AN responses show that tonotopic coding of broadband noise is maintained at stimulus levels up to at least $\sim 80$ dB SPL, even in low-threshold AN fibers with high spontaneous discharge rates (Recio-Spinoso et al., 2005; Henry et al., 2016). These studies found small downward BF shifts in AN suprathreshold coding with increasing stimulus level $(\sim 5 \%$ decrease in BF from 45 to $80 \mathrm{~dB}$ SPL in Fig. 14 of Recio-Spinoso et al., 2005) and broader tuning bandwidth, but no abnormal response to low-frequency stimulus components as occurred here with hearing loss. Thus, the coding deficits observed with moderate to severe MHL and with mild NIHL do not simply reflect the higher stimulus levels used to evoke these responses.

Overall, our findings provide physiological support for diagnostic efforts to dissect the single clinical category of SNHL based on different etiologies, which may be possible based on audiogram configuration (Dubno et al., 2013). To the extent that speech intelligibility depends on robust tonotopic encoding of TFS and ENV cues (Lorenzi et al., 2006; Jørgensen et al., 2013), our results comparing MHL and NIHL effects between AN fibers at equal threshold elevation suggest that differences in speech perception among individuals with similar audiograms may (at least partially) reflect differences in peripheral coding associated with specific cochlear pathologies. The less severe effects of MHL here are consistent with general findings from human temporal bone studies in which patients with strial presbycusis (metabolic hearing loss) may retain excellent word discrimination scores (Schuknecht and Gacek, 1993). Behavioral tests with lowfrequency maskers, which are predicted based on our results to disproportionately impair perception in cases of NIHL, may be useful for diagnosing the extent of NIHL versus MHL in the clinic.

Hearing aids are currently fit based on individual frequency thresholds in the audiogram without explicitly factoring in the across-frequency configuration that has proven to be a successful basis for classifying NIHL and MHL etiologies in animal models (Schmiedt et al., 2002; Schmiedt, 2010) and in humans (Dubno et al., 2013). Our results highlighting the importance of the TTR in accounting for the coding of complex sounds provide physiological reasons that current hearing aid fitting approaches may be inadequate. Different signal-processing strategies are needed to address fundamentally different peripheral coding deficits in individuals with the same degree of hearing loss but different cochlear pathologies (i.e., MHL vs NIHL). Successful strategies may be particularly difficult to develop for individuals with NIHL given the profound tonotopic distortion of AN temporal coding demonstrated here. Finally, our results have important implications for public health awareness given the increasing prevalence of NIHL and the important fact that it is preventable.

\section{References}

Bates D, Mächler M, Bolker B, Walker S (2015) Fitting linear mixed-effects models using lme4. J Stat Softw 67:1-48.

Bharadwaj HM, Masud S, Mehraei G, Verhulst S, Shinn-Cunningham BG (2015) Individual differences reveal correlates of hidden hearing deficits. J Neurosci 35:2161-2172.

Chintanpalli A, Heinz MG (2007) Effect of auditory nerve response variability on estimates of tuning curves. J Acoust Soc Am 122:EL203-EL209.

Dubno JR, Dirks DD, Langhofer LR (1982) Evaluation of hearing-impaired listeners using a nonsense-syllable test. II. Syllable recognition and consonant confusion patterns. J Speech Hear Res 25:141-148.
Dubno JR, Eckert MA, Lee FS, Matthews LJ, Schmiedt RA (2013) Classifying human audiometric phenotypes of age-related hearing loss from animal models. J Assoc Res Otolaryngol 14:687-701.

Eggermont JJ, Johannesma PM, Aertsen AM (1983) Reverse-correlation methods in auditory research. Q Rev Biophys 16:341-414.

Fitzgibbons PJ, Gordon-Salant S (2010) Behavioral studies with aging humans: hearing sensitivity and psychoacoustics. In: The aging auditory system (Gordon-Salant S, Frisina RD, Fay RR, Popper A, eds), pp 111134. New York: Springer.

Halpin C, Rauch SD (2009) Clinical implications of a damaged cochlea: pure tone thresholds vs information-carrying capacity. Otolaryngol Head Neck Surg 140:473-476.

He NJ, Schmiedt RA (1996) Effects of aging on the fine structure of the 2f1-f2 acoustic distortion product. J Acoust Soc Am 99:1002-1015.

Heinz MG, Henry KS (2013) Modeling disrupted tonotopicity of temporal coding following sensorineural hearing loss. Proc Mtgs Acoust 19:050177.

Heinz MG, Colburn HS, Carney LH (2001) Evaluating auditory performance limits: i. one-parameter discrimination using a computational model for the auditory nerve. Neural Comput 13:2273-2316.

Henry KS, Kale S, Heinz MG (2016) Distorted tonotopic coding of temporal envelope and fine structure with noise-induced hearing loss. J Neurosci 36:2227-2237.

Humes LE, Dubno JR, Gordon-Salant S, Lister JJ, Cacace AT, Cruickshanks KJ, Gates GA, Wilson RH, Wingfield A (2012) Central presbycusis: a review and evaluation of the evidence. J Am Acad Audiol 23:635-666.

Johnson DH (1980) The relationship between spike rate and synchrony in responses of auditory-nerve fibers to single tones. J Acoust Soc Am 68: $1115-1122$.

Jørgensen S, Ewert SD, Dau T (2013) A multi-resolution envelopepower based model for speech intelligibility. J Acoust Soc Am 134: $436-446$

Joris PX, Yin TC (1992) Responses to amplitude-modulated tones in the auditory nerve of the cat. J Acoust Soc Am 91:215-232.

Lewis ER, van Dijk P (2004) New variation on the derivation of spectrotemporal receptive fields for primary auditory afferent axons. Hear Res 189:120-136.

Lewis ER, Henry KR, Yamada WM (2002) Tuning and timing in the gerbil ear: Wiener-kernel analysis. Hear Res 174:206-221.

Liberman MC (1982) The cochlear frequency map for the cat: labeling auditory nerve fibers of known characteristic frequency. J Acoust Soc Am 72:1441-1449.

Liberman MC (1984) Single-neuron labeling and chronic cochlear pathology. I. Threshold shift and characteristic-frequency shift. Hear Res 16:3341.

Liberman MC, Dodds LW (1984) Single-neuron labeling and chronic cochlear pathology. III. Stereocilia damage and alterations of threshold tuning curves. Hear Res 16:55-74.

Lopez-Poveda EA, Johannesen PT (2012) Behavioral estimates of the contribution of inner and outer hair cell dysfunction to individualized audiometric loss. JARO - J Assoc Res Otolaryngol 13:485-504.

Lorenzi C, Gilbert G, Carn H, Garnier S, Moore BC (2006) Speech perception problems of the hearing impaired reflect inability to use temporal fine structure. Proc Natl Acad Sci U S A 103:18866-18869.

Lurie MH, Davis H, Hawkins JE (1944) Acoustic trauma of the organ of Corti in the guinea pig. Laryngoscope 54:375-386.

Mills DM, Rubel EW (1994) Variation of distortion product otoacoustic emissions with furosemide injection. Hear Res 77:183-199.

Mills DM, Norton SJ, Rubel EW (1993) Vulnerability and adaptation of distortion product otoacoustic emissions to endocochlear potential variation. J Acoust Soc Am 94:2108-2122.

Mills JH, Schmiedt RA, Schulte BA, Dubno JR (2006) Age-related hearing loss: a loss of voltage, not hair cells. Semin Hear 27:228-236.

Moore BC, Vickers DA, Plack CJ, Oxenham AJ (1999) Inter-relationship between different psychoacoustic measures assumed to be related to the cochlear active mechanism. J Acoust Soc Am 106:2761-2778.

Recio-Spinoso A, Temchin AN, van Dijk P, Fan YH, Ruggero MA (2005) Wiener-kernel analysis of responses to noise of chinchilla auditory nerve fibers. J Neurophysiol 93:3615-3634.

Ruggero MA, Rich NC (1991) Furosemide alters organ of corti mechanics: evidence for feedback of outer hair cells upon the basilar membrane. J Neurosci 11:1057-1067. 
Ruggero MA, Robles L, Rich NC (1992) Two-tone suppression in the basilar membrane of the cochlea: mechanical basis of auditory nerve rate suppression. J Neurophysiol 68:1087-1099.

Rybak LP, Whitworth C, Weberg A, Scott V (1992) Effects of organic acids on the edema of the stria vascularis induced by furosemide. Hear Res 59:75-84.

Sayles M, Heinz MG (2017) Afferent coding and efferent control in the normal and impaired cochlea. In: Understanding the cochlea (Manley GA, Gummer AW, Popper AN, Fay RR, eds), pp 215-252. Cham, Switzerland: Springer.

Schmiedt RA (2010) The physiology of cochlear presbycusis. In: The aging auditory system (Gordon-Salant S, Frisina RD, Fay RR, Popper A, eds), pp 9-38. New York: Springer.

Schmiedt RA, Mills JH, Adams JC (1990) Tuning and suppression in auditory nerve fibers of aged gerbils raised in quiet or noise. Hear Res 45:221-236.
Schmiedt RA, Lang H, Okamura HO, Schulte BA (2002) Effects of furosemide applied chronically to the round window: A model of metabolic presbyacusis. J Neurosci 22:9643-9650.

Schuknecht HF, Gacek MR (1993) Cochlear pathology in presbycusis. Ann Otol Rhinol Laryngol 102:1-16.

Sewell WF (1984) The effects of furosemide on the endocochlear potential and auditory nerve fiber tuning curves in cats. Hear Res 14:305-314.

Temchin AN, Rich NC, Ruggero MA (2008) Threshold tuning curves of chinchilla auditory nerve fibers. I. Dependence on characteristic frequency and relation to the magnitudes of cochlear vibrations. J Neurophysiol 100:2889-2898.

van Dijk P, Wit HP, Segenhout JM, Tubis A (1994) Wiener kernel analysis of inner ear function in the American bullfrog. J Acoust Soc Am 95:904-919.

van Drongelen W (2010) Wiener series. In: Signal processing for neuroscientists, a companion volume: Advanced topics, nonlinear techniques and multi-channel analysis, pp 61-90. Amsterdam: Elsevier. 